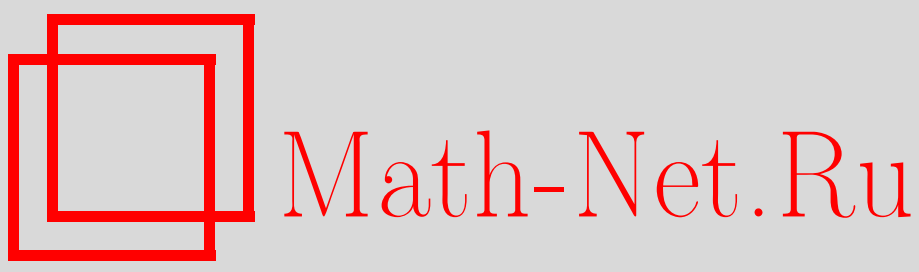

Г. Б. Клейнер, Поливекторы ранга 2 над полями и коммутативными кольцами, Матем. сб., 2002, том 193, номер 5, 77-94

DOI: https://doi.org/10.4213/sm652

Использование Общероссийского математического портала Math-Net.Ru подразумевает, что вы прочитали и согласны с пользовательским соглашением http://www . mathnet.ru/rus/agreement

Параметры загрузки:

IP: 54.84 .234 .179

26 апреля 2023 г., $07: 28: 40$ 
УДК 512.552

\author{
Г.Б. Клейнер
}

\title{
Поливекторы ранга 2 над полями и коммутативными кольцами
}

\begin{abstract}
В работе изучается следующий вопрос: когда однородньй элемент грассмановой алгебры представим в виде суммы двух разложимых элементов? Для внешней алгебры над полем получены необходимые и достаточные условия такого представления, над произвольным челостным кольцом-ряд необходимых условий, а над кольцами Крулля - также и ряд достаточных условий. В частности, установлено, что единственньпи кольцами, над которыми проверка 2-разложимости проводится так же, как над полями, являются поля, т.е. "2-плюккеровых" колец не существует.

Библиография: 11 названий.
\end{abstract}

\section{§1. Введение}

Пусть $A$ - коммутативное кольцо с единицей, $A^{n}$ - свободньй $A$-модуль ранга $n$ с базисом $e_{1}, \ldots, e_{n}$ и $\Lambda^{m}\left(A^{n}\right)$ - его внешняя степень. Совокупность внешних произведений $\left\{e_{i_{1}} \wedge \cdots \wedge e_{i_{m}}\right\}, 1 \leqslant i_{1}<\cdots<i_{m} \leqslant n$, базисных элементов модуля $A^{n}$ образует базис свободного $A$-модуля $\Lambda^{m}\left(A^{n}\right)$, поэтому всякий $m$-вектор $\omega \in \Lambda^{m}\left(A^{n}\right)$ однозначно представим в виде

$$
\omega=\sum_{1 \leqslant i_{1}<\cdots<i_{m} \leqslant n} a_{i_{1} \ldots i_{m}} e_{i_{1}} \wedge \cdots \wedge e_{i_{m}}
$$

где $a_{i_{1} \ldots i_{m}} \in A$.

Во многих случаях, однако, удобнее записьвать $\omega$ не в виде линейной комбинации элементов индуцированного базиса $e_{i_{1}} \wedge \cdots \wedge e_{i_{m}}$ (такое представление, конечно, зависит от выбора базиса $e_{1}, \ldots, e_{n}$ в $\left.A^{n}\right)$, а в виде суммы наименьшего числа произвольных разложимых $m$-векторов

$$
\omega=x_{1}^{1} \wedge \cdots \wedge x_{m}^{1}+\cdots+x_{1}^{k} \wedge \cdots \wedge x_{m}^{k}
$$

Это представление (оно называется минимальным разложением поливекто$p a$, а число слагаемых $k$ - его рангом) позволяет вычислить ряд важных инвариантов $m$-вектора, в частности найти размерность наименьшего подпространства (в случае, когда $A$ - поле), $m$-я степень которого содержит данный поливектор.

Настоящая работа посвящена вопросам вычисления ранга и минимального разложения поливекторов, в основном над полями и кольцами Крулля.

(C) Г.Б. КлейнеР 2002 
Первые результаты, касающиеся определения ранга $m$-векторов, принадлежат Плюккеру [1] и Сегре [2]. Они доказали, что в случае поля необходимьм и достаточным условием разложимости $m$-вектора $\omega \in \Lambda^{m}\left(A^{n}\right)$ является выполнение так называемых соотношений Плюккера

$$
\sum_{s=0}^{m}(-1)^{s} a_{i_{1} \ldots i_{m-1} j_{s}} a_{j_{0} \ldots j_{s-1} j_{s+1} \ldots j_{m}}=0, \quad 1 \leqslant i_{1}, \ldots, i_{m-1}, j_{0}, \ldots, j_{m} \leqslant n
$$

между его координатами. Найти аналогичные соотношения, выделяющие класс $m$-векторов ранга не больше $k$ при $k>1$, удавалось лишш для $m=2$ [3]. В 1973 г. Буземан и Глазго доказали [4], что если $A=\mathbb{R}$ или $\mathbb{C}$, то множество $m$-векторов ранга $\leqslant k$ при $k>1, m>2$ не является ни алгебраическим, ни даже замкнутым множеством, следовательно, аналога соотношений Плюккера в этом случае нет. Известно, однако, что соотношения (3) эквивалентны условию (см. [5])

$$
\operatorname{rank} B_{0}(\omega)=m,
$$

где $B_{0}(\omega)=\left\{x \in A^{n}: x \wedge \omega=0\right\}$.

Поэтому естественно пытаться охарактеризовать класс $k$-разложимых $m$-векторов при $k>1$ с помощью аналогичных (4) условий на некоторые связанные с $m$-вектором и в принципе вычислимые модули.

Для $k=2$ и произвольного $m$ соответствующий результат анонсирован автором [6]. Именно пусть $E_{1}(\omega)$ - подпространство, порожденное теми $x \in A^{n}$, для которых $\operatorname{rank}(x \wedge \omega) \leqslant 1$. Тогда для 2 -разложимости $\omega$ необходимо и достаточно, чтобы

$$
\operatorname{dim} B_{0}(\omega)+\operatorname{dim} E_{1}(\omega)=2 m
$$

При $k>2, m>2$ вопрос об условиях, необходимых и достаточных для $k$-разложимости $m$-вектора, остается открытым даже в случае полей.

Пусть теперь $A$ - целостное коммутативное кольцо. Естественное вложение $A \subset \operatorname{Fr}(A)$ кольца $A$ в его поле частных позволяет рассматривать всякий $m$-вектор $\omega \in \Lambda^{m}\left(A^{n}\right)$ как элемент $\Lambda^{m}\left(\operatorname{Fr}(A)^{n}\right)$; при этом, очевидно, разложимый над $A$ $m$-вектор разложим и над $\operatorname{Fr}(A)$. Следовательно, соотношения Плюккера (и эквивалентное им условие (4)), а также равенство (5) являются необходимьми условиями соответственно для 1- и 2-разложимости.

Таубер [7] рассматривал кольца, в которых соотношения Плюккера являются также и достаточными для разложимости (эти кольца получили название плюккеровых). Было доказано, что такими являются одно- и двумерные локальные кольца, дедекиндовы кольца и кольцо многочленов от одной переменной над дедекиндовым кольцом. Среди ненётеровых плюккеровых колец можно назвать кольца Безу.

Таким образом, сушествует широкий класс колец, для которых проверка 1-разложимости поливектора проводится так же, как и в полях (например, с помощью соотношений (3) или (4)). В настояшей работе доказывается теорема 4.1, согласно 
которой эта ситуация не продолжается на случай 2-разложимости: единственными кольцами, в которых множество 2-разложимых $m$-векторов над кольцом совпадает с множеством 2-разложимых $m$-векторов над полем частных ("2-плюккеровы кольца"), являются поля. Поэтому при $k=2$ особый интерес представляют условия "индивидуальной" $k$-разложимости $m$-векторов над кольцом, специфические для данного класса колец и дополняющие необходимые условия 2-разложимости (5).

Для $k=1$ в этом направлении известны следующие результаты.

Теорема 1.1 [8]. Пусть $A$ - коммутативное кольио, $\omega \in \Lambda^{m}\left(A^{n}\right)-m$-вектор. Если $B_{0}(\omega)$ - свободный модуль ранга $m$, mо $\operatorname{rank} \omega=1$.

Поливектор $\omega$ называется примитивным, если дивизор идеала, порожденньй его координатами, равен 1.

Tеорема 1.2 [9]. Пусть $A$ - кольио Крулля, $\omega$ - примитивный Для того чтобы $\operatorname{rank} \omega=1$, необходимо и достаточно, чтобъ $B_{0}(\omega)$ бъл свободным А-модулем ранга $\mathrm{m}$.

В настоящей работе получены аналогичные результаты (теоремы 5.1 и 5.2 ) для $k=2$.

Работа состоит из пяти параграфов.

В $\S 2$ исследуется множество $B_{1}(\omega)=\left\{x \in A^{n}: \operatorname{rank}(x \wedge \omega) \leqslant 1\right\}$ для $m$-векторов ранга 2 и устанавливается его связь с подпространствами, порожденньпи векторами минимального разложения $\omega$. Этот результат используется в $\S 3$ для вывода теоремы единственности минимального разложения $m$-векторов ранга 2. Параграф 4 содержит полное доказательство необходимого и достаточного условия (5) 2-разложимости поливекторов вида $\omega=x_{1} \wedge \cdots \wedge x_{m-2} \wedge \omega^{\prime}$, где $x_{i} \in A$, $\omega^{\prime} \in \Lambda^{2}\left(A^{n}\right)$, над плюккеровьми кольцами. Здесь же доказывается теорема несуществования 2-плюккеровых колец. Параграф 5 посвящен доказательству условий 2 -разложимости $m$-векторов, не представимых в виде $\omega=x_{1} \wedge \cdots \wedge x_{m-2} \wedge \omega^{\prime}$, где $\omega^{\prime}-2$-вектор, над кольцами Крулля.

Фиксируем следующие обозначения: $A$ - целостное коммутативное кольцо; $\operatorname{Fr}(A)$ - его поле частных; $A^{n}$ - свободный $A$-модуль ранга $n$ с образующими $e_{1}, \ldots, e_{n} ; M$ - подмодуль в $A^{n} ; \Lambda^{m}(M)$ - образ в $\Lambda^{m}\left(A^{n}\right) m$-й внешней степени модуля $M$ при отображении $\Lambda^{m} \varepsilon$, где $\varepsilon-$ вложение $M$ в $A^{n} ; \omega-$ $m$-вектор из $\Lambda^{m}\left(A^{n}\right) ; \widehat{x}$ - внешнее произведение элементов $x_{1}, \ldots, x_{n} \in A^{n}$; $X$ - порожденный ими подмодуль; $\left\{a_{1}, \ldots, a_{n}\right\}$ - множество, состоящее из элементов $a_{1}, \ldots, a_{n} ; B_{0}(\omega)=\left\{x \in A^{n}: x \wedge \omega=0\right\} ; b_{0}(\omega)=\operatorname{rank} B_{0}(\omega)$; $B_{1}(\omega)=\left\{x \in A^{n}: x \wedge \omega-\right.$ плюккеров $(m+1)$-вектор $\} ; b_{1}(\omega)=\operatorname{rank} B_{1}(\omega)$; $E_{0}(\omega)=B_{0}(\omega) ; E_{1}(\omega)$ - подмодуль в $A^{n}$, порожденньй множеством $B_{1}(\omega)$; $\omega_{1} \| \omega_{2}-$ сушествует ненулевой элемент $\alpha \in \operatorname{Fr}(A)$, для которого $\alpha \omega_{1}=\omega_{2}$ $\left(\omega_{1}, \omega_{2} \in \Lambda^{m}\left(A^{n}\right)\right) ; \operatorname{Supp} \omega=\inf \left\{M-\right.$ подмодуль в $\left.A^{n}: \omega \in \Lambda^{m}(M)\right\} ; \widetilde{M}=$ $\inf \left\{M^{\prime}\right.$ - подмодуль в $A^{n}: M \subset M^{\prime}, A^{n} / M^{\prime}$ не имеет кручения $\} ; \operatorname{div} \omega-$ дивизор идеала, порожденного координатами $\omega$. 


\section{§2. Строение множества $B_{1}(\omega)$}

Лемма 2.1 [10; теорема 4]. Пусть $A$ - поле, $\omega \in \Lambda^{m}\left(A^{n}\right)-m$-вектор ранга $k$, $\omega=\widehat{x}_{1}+\cdots+\widehat{x}_{k}$. Тогда $B_{0}(\omega)=X_{1} \cap \cdots \cap X_{k}$.

Другими словами, всякий т-вектор $\omega$ с $b_{0}(\omega) \neq 0$ представим в виде $\omega=b \wedge \omega^{\prime}$, где $b$ - произведение всех векторов какого-то базиса пространства $B_{0}(\omega)$, a $\omega^{\prime}-\left(m-b_{0}(\omega)\right)$-вектор того же ранга, что и $\omega$, причем $b_{0}\left(\omega^{\prime}\right)=0$.

Если $A$ - поле, $\omega \in \Lambda^{m}\left(A^{n}\right)$, то носитель $m$-вектора $\omega(\operatorname{Supp} \omega)$, очевидно, существует и единственен.

Лемма 2.2 [6; лемма 1]. Пусть $A-$ поле, $\omega \in \Lambda^{m}\left(A^{n}\right)-m$-вектор ранга $k$, $\omega=\widehat{x}_{1}+\cdots+\widehat{x}_{k}$. Тогдa $\operatorname{Supp} \omega=X_{1}+\cdots+X_{k}=E_{k-1}(\omega)$.

Отсюда следует

Лемма 2.3. Пусть $A-$ поле, $\omega \in \Lambda^{m}\left(A^{n}\right), y_{1}, \ldots, y_{s}$ - линейно независимые векторы из $A^{n}$, причем порожденное ими подпространство $Y$ имеет нулевое пересечение $c \operatorname{Supp} \omega$. Тогда $\operatorname{rank}\left(y_{1} \wedge \cdots \wedge y_{s} \wedge \omega\right)=\operatorname{rank} \omega$.

ПреДЛОЖЕНИЕ 2.1. Пусть $A-$ поле, $\omega \in \Lambda^{m}\left(A^{n}\right)-m$-вектор ранга 2, $\omega=\widehat{x}_{1}+\widehat{x}_{2}$. Тогда если $b_{0}(\omega)=m-2$, то $B_{1}(\omega)=X_{1}+X_{2}$, в противном cлучае $B_{1}(\omega)=X_{1} \cup X_{2}$.

ДокАЗАТЕЛЬСтво. Пусть $\operatorname{rank} \omega=2$. По лемме 1.1 можно считать

$$
\omega=w_{1} \wedge \cdots \wedge w_{b_{0}} \wedge\left(v_{1}^{1} \wedge \cdots \wedge v_{m-b_{0}}^{1}+v_{1}^{2} \wedge \cdots \wedge v_{m-b_{0}}^{2}\right)
$$

где векторы $w_{i}, v_{j}^{1}, v_{k}^{2}$ линейно независимы в совокупности.

Пусть $z \in B_{1}(\omega)$. Так как $B_{1}(\omega) \subset \operatorname{Supp} \omega=X_{1}+X_{2}$, то $z=z_{\omega}+z_{1}+z_{2}$, где $z_{\omega}$ лежит в линейном пространстве $B_{0}(\omega)$ с базисом $\left\{w_{i}\right\}$, а $z_{j}, j=1,2$, лежит в линейном пространстве, порожденном $\left\{v_{k}^{j}\right\}$. Имеем:

$$
z_{\omega}= \pm \widehat{w} \wedge\left(z_{1} \wedge \widehat{v}^{1}+z_{2} \wedge \widehat{v}^{2}\right)
$$

Поскольку этот поливектор должен иметь единичный ранг, то единичньй ранг должен иметь и вектор $z_{1} \wedge \widehat{v}^{1}+z_{2} \wedge \widehat{v}^{2}$, откуда получаем равенство

$$
B_{1}(\omega)=A w \oplus B_{1}\left(\widehat{v}^{1}+\widehat{v}^{2}\right) .
$$

Теперь вопрос сводится к изучению векторов $\omega$ вида $\omega=\widehat{v}^{1}+\widehat{v}^{2}$, т.е. к случаю $b_{0}=0$. Достаточно доказать, что для такого $\omega$

$$
B_{1}(\omega)= \begin{cases}A v^{1}+A v^{2}, & m=2, \\ A v^{1} \cup A v^{2}, & m \neq 2 .\end{cases}
$$

При $m=0,1$ поливектор $\omega$ нулевой или плюккеров, и утверждение очевидно. 
Пусть $m \geqslant 2$. Легко проверить непосредственно, что $X_{1} \cup X_{2} \subset B_{0}(\omega)$. Предположим, что $z$-произвольный вектор из $X_{1}+X_{2}$, не лежащий в $X_{1} \cup X_{2}$. С точностью до замены базиса в пространствах $X_{1}$ и $X_{2}$ можно считать $z_{1}=v_{1}^{1}$ и $z_{2}=v_{1}^{2}$. Тогда при $m=2$ получаем:

$$
z \wedge \omega=z_{1} \wedge \widehat{v}^{1}+z_{2} \wedge \widehat{v}^{2}=v_{1}^{1} \wedge v_{1}^{2} \wedge v_{2}^{2}-v_{1}^{1} \wedge v_{1}^{2} \wedge v_{2}^{1}=v_{1}^{1} \wedge v_{1}^{2} \wedge\left(v_{2}^{2}-v_{2}^{1}\right),
$$

т.е. $X_{1}+X_{2} \subset B_{0}(\omega)$. Если же $m>2$, то

$$
z \wedge \omega=v_{1}^{1} \wedge v_{1}^{2} \wedge\left(v_{2}^{2} \wedge \cdots \wedge v_{m}^{2}-v_{2}^{1} \wedge \cdots \wedge v_{m}^{1}\right)
$$

т.е. $z \wedge \omega-$ не плюккеров вектор.

ЗАмЕчАНИЕ. Отметим один из пунктов доказательства: если rank $\omega=2$, то $b_{0}(\omega) \leqslant m-2$.

Пусть теперь $A$ - произвольное коммутативное кольцо, $\omega \in \Lambda^{m}\left(A^{n}\right)$. В этом случае множество $c_{1}(\omega)=\left\{x \in A^{n}: x \wedge \omega\right.$ - разложимый $(m+1)$-вектор $\}$ не совпадает, вообще говоря, с множеством $B_{1}(\omega)=\left\{x \in A^{n}: x \wedge \omega\right.$ - плюккеров $(m+1)$-вектор $\}$. Однако если $b_{0}(\omega)<m-2$, то эти множества одинаковы.

СлеДСТВИЕ 2.1. Пусть $A-$ коммутативное кольцо, $\omega \in \Lambda^{m}\left(A^{n}\right)-m$-вектор ранга 2 , причем $b_{0}(\omega)<m-2$. Тогда $B_{1}(\omega)=c_{1}(\omega)=\widetilde{X}_{1} \cap \widetilde{X}_{2}$.

ДокаЗАтельство. Рассмотрим $\omega$ как вектор над $\operatorname{Fr}(A)$ и обозначим через $\operatorname{rank}_{\mathrm{Fr}} \omega$ его ранг как элемента $\Lambda^{m}\left(\operatorname{Fr}(A)^{n}\right)$. Пусть $B_{1}^{\mathrm{Fr}}(\omega)=\left\{x \in A^{n}: x \wedge \omega-\right.$ плюккеров $(m+1)$-вектор в $\left.\Lambda^{m+1}\left(\operatorname{Fr}(A)^{n}\right)\right\}$. Тогда $B_{1}(\omega)=B_{1}^{\mathrm{Fr}}(\omega) \cap A^{n}$. Если $\operatorname{rank}_{\mathrm{Fr}} \omega=1$, то $b_{0}(\omega)=m$, что противоречит условию. Следовательно, $\operatorname{rank}_{\mathrm{Fr}} \omega=2$. В силу предложения $2.1 B_{1}^{\mathrm{Fr}}(\omega)=\operatorname{Fr} X_{1} \cup \operatorname{Fr} X_{2}$, где $\operatorname{Fr} X_{i}$ - подпространство, порожденное в $\operatorname{Fr}(A)^{n}$ векторами разложения $\widehat{x}_{i}, i=1,2$. Значит, $B_{1}(\omega)=B_{1}^{\operatorname{Fr}}(\omega) \cap A^{n}=\left(\operatorname{Fr} X_{1} \cap A^{n}\right) \cup\left(\operatorname{Fr} X_{1} \cap A^{n}\right)=\widetilde{X}_{1} \cup \widetilde{X}_{2}$.

Далее, $c_{1}(\omega)$ всегда лежит в $B_{1}(\omega)$. Пусть $x \in B_{1}(\omega)$. Тогда $x \in \widetilde{X}_{1} \cup \widetilde{X}_{2}$; предположим, что $x \in \widetilde{X}_{1}$. Тогда $x=y / a$, где $y \in X_{1}, a \in A, a \neq 0$. Теперь $x \wedge w=x \wedge \widehat{x}_{2}-$ разложимый $(m+1)$-вектор. Таким образом, $B_{1}(\omega) \subset c_{1}(\omega)$ и, следовательно, $B_{1}(\omega)=c_{1}(\omega)=\widetilde{X}_{1} \cup \widetilde{X}_{2}$.

\section{§ 3. Единственность минимального разложения $m$-векторов ранга 2}

Пусть $\omega-m$-вектор ранга $k$ над коммутативным кольцом. Скажем, что минимальное разложение $\omega$ единственно, если из равенств

$$
\omega=\widehat{x}_{1}+\cdots+\widehat{x}_{k}=\widehat{y}_{1}+\cdots+\widehat{y}_{k},
$$

где $\widehat{x}_{1}, \ldots, \widehat{x}_{k}, \widehat{y}_{1}, \ldots, \widehat{y}_{k}$ - разложимые $m$-векторы, вытекает, что $\widehat{y}_{\pi(i)}=\widehat{x}_{i}$, $i=1, \ldots, k$, для некоторой перестановки $\pi$ на множестве $\{1,2, \ldots, k\}$.

Tеорема 3.1. Пусть $A-$ поле, $\omega \in \Lambda^{m}\left(A^{n}\right)-m$-вектор ранга 2. Тогда следующие условия әквивалентны:

(a) $b_{0}(\omega) \neq m-2$;

(б) минимальное разложение поливектора $\omega$ единственно. 
ДокАЗАТЕльство. Как следует из леммы 2.1, всякое минимальное разложение имеет вид $\omega=\widehat{w}_{0} \wedge\left(\widehat{w}_{1}+\widehat{w}_{2}\right)$, где $\widehat{w}_{0}-$ произведение каких-то базисных векторов пространства $B_{0}(\omega)$, а все компоненты поливекторов $\widehat{w}_{1}, \widehat{w}_{2}$ линейно независимы и не лежат в $B_{0}(\omega)$. Поскольку $\widehat{w}_{0}$ определяется однозначно с точностью до скалярного множителя, достаточно доказывать единственность (или не единственность) второго сомножителя, т.е. рассматривать случай вектора $\omega$, для которого $b_{0}(\omega)=0$.

Пусть $m=2$. Тогда $\omega=w_{1}^{1} \wedge w_{2}^{1}+w_{1}^{2} \wedge w_{2}^{2}=w_{1}^{1} \wedge\left(w_{2}^{1}+w_{2}^{2}\right)+\left(w_{1}^{2}-w_{1}^{1}\right) \wedge w_{2}^{2}$. Из линейной независимости компонент поливекторов $\widehat{w}_{1}, \widehat{w}_{2}$ получаем, что разложение неединственно.

Пусть теперь $m>2$. Предположим, что $\omega=\widehat{w}_{1}+\widehat{w}_{2}=\widehat{v}_{1}+\widehat{v}_{2}$. Имеем:

$$
0=B_{0}(\omega)=\operatorname{Supp} \widehat{w}_{1} \cap \operatorname{Supp} \widehat{w}_{2}=\operatorname{Supp} \widehat{v}_{1} \cap \operatorname{Supp} \widehat{v}_{2}
$$

но по предложению 1.1

$$
B_{1}(\omega)=\operatorname{Supp} \widehat{w}_{1} \cup \operatorname{Supp} \widehat{w}_{2}=\operatorname{Supp} \widehat{v}_{1} \cup \operatorname{Supp} \widehat{v}_{2},
$$

откуда с точностью до перестановки слагаемых получаем, что $\widehat{v}_{1}=\alpha \widehat{w}_{1}, \widehat{v}_{2}=\beta \widehat{w}_{2}$, где $\alpha, \beta \in A$. Из линейной независимости поливекторов $\widehat{w}_{1}, \widehat{w}_{2}$ получаем, что $\alpha=\beta=1$.

СЛЕДСТВИЕ 3.1. Пусть $A$ - произвольное коммутативное целостное кольчо, $\omega \in \Lambda^{m}\left(A^{n}\right)$ - m-вектор ранга 2 . Если $b_{0}(\omega) \neq m-2$, то минимальное разложсение $\omega$ единственно.

ДоказАтельство. Пусть $\omega=\widehat{x}_{1}+\widehat{x}_{2}=\widehat{y}_{1}+\widehat{y}_{2}$, где $\widehat{x}_{i}, \widehat{y}_{i}-$ разложимые $m$-векторы, $i=1,2$. Вложив $\Lambda^{m}\left(A^{n}\right)$ в $\Lambda^{m}\left(\mathrm{Fr}^{n}\right)$, получим два разложения $m$-вектора над полем $\operatorname{Fr}(A)$. Применяя теорему 3.1 и учитывая, что ранг $B_{0}(\omega)$ равен размерности пространства $\left\{x \in \mathrm{Fr}^{n}: x \wedge \omega=0\right\}$, заключаем, что эти разложения совпадают в $\Lambda^{m}\left(\mathrm{Fr}^{n}\right)$, а следовательно, и в $\Lambda^{m}\left(A^{n}\right)$.

Лемма 3.1. Пусть $P_{1}, P_{2}, Q_{1}, Q_{2}$ - әруппь, причем ни одно из множеств $P_{1}, P_{2}$ и $Q_{1}, Q_{2}$ не лежит в другом. Если $P_{1} \cup P_{2}=Q_{1} \cup Q_{2}$, то $P_{i}=Q_{\pi(i)}$ для некоторой перестановки $\pi$ на множестве $\{1,2\}$.

Из предложения 2.1 и теоремы 3.1 видно, что множества $B_{0}(\omega)$ и $B_{1}(\omega)$ несут довольно полную информацию о минимальном разложении $m$-вектора $\omega$ ранга 2 . Точная формулировка соответствующего результата такова.

ПРЕДЛОЖЕНИЕ 3.1. Пусть $A-$ коммутативное кольцо, $\omega=\widehat{x}_{1}+\widehat{x}_{2}, \omega^{\prime}=$ $\widehat{x}_{1}^{\prime}+\widehat{x}_{2}^{\prime}-$-векторьи ранга 2 , причем $\operatorname{rank} B_{0}(\omega)<m-2$. Следующие условия әквивалентны:

(a) $\widehat{x}_{i} \| \widehat{x}_{\pi(i)}^{\prime}$, где $\pi$ - некоторая перестановка на множестве $\{1,2\}$;

(б) $B_{1}(\omega)=B_{1}\left(\omega^{\prime}\right)$;

(в) $B_{0}(\omega)=B_{0}\left(\omega^{\prime}\right), B_{1}(\omega)=B_{1}\left(\omega^{\prime}\right)$. 
ДокАЗАтЕльСтво. (а) $\Rightarrow\left(\right.$ б). Если $\widehat{x}_{i} \| \widehat{x}_{\pi(i)}^{\prime}$, то $\widetilde{X}_{i}=\widetilde{X}_{\pi(i)}^{\prime}, i=1,2$. Поэтому $B_{0}(\omega)=\widetilde{X}_{1} \cap \widetilde{X}_{2}=\widetilde{X}_{1}^{\prime} \cap \widetilde{X}_{2}^{\prime}=B_{0}\left(\omega^{\prime}\right)\left(\right.$ лемма 2.1), $B_{1}(\omega)=\widetilde{X}_{1} \cup \widetilde{X}_{2}=\widetilde{X}_{1}^{\prime} \cup$ $\widetilde{X}_{2}^{\prime}=B_{1}\left(\omega^{\prime}\right)$ (следствие 2.1).

$\left(\right.$ б) $\Rightarrow\left(\right.$ a). $B_{1}(\omega)=\widetilde{X}_{1} \cup \widetilde{X}_{2}=B_{1}\left(\omega^{\prime}\right)=\widetilde{X}_{1}^{\prime} \cup \widetilde{X}_{2}^{\prime}$. Так как $\operatorname{rank} \widetilde{X}_{i}=\operatorname{rank} \widetilde{X}_{i}^{\prime}=$ $m, i=1,2$, то из включения $\widetilde{X}_{1} \subset \widetilde{X}_{2}$ (или из включения $\widetilde{X}_{1}^{\prime} \subset \widetilde{X}_{2}^{\prime}$ ) следовало бы, что $\widetilde{X}_{1}=\widetilde{X}_{2}$ (соответственно $\widetilde{X}_{1}^{\prime}=\widetilde{X}_{2}^{\prime}$ ). В свою очередь, это означает, что $\widehat{x}_{1} \| \widehat{x}_{2}$ (или $\left.\widehat{x}_{1}^{\prime} \| \widehat{x}_{2}^{\prime}\right)$. Но тогда $\operatorname{rank} B_{0}(\omega)=\operatorname{rank} B_{0}\left(\omega^{\prime}\right)=m$, что противоречит условию. Следовательно, к модулям $\widetilde{X}_{1}, \widetilde{X}_{2}, \widetilde{X}_{1}^{\prime}, \widetilde{X}_{2}^{\prime}$ применима лемма 3.1 , в силу которой $\widetilde{X}_{i}=\widetilde{X}_{\pi(i)}^{\prime}$ для некоторой перестановки $\pi$ на множестве $\{1,2\}$. Отсюда $\widehat{x}_{i} \| \widehat{x}_{\pi(i)}^{\prime}$ для $i=\{1,2\}$.

(в) $\Rightarrow($ б). Очевидно.

$\left(\right.$ a) $\Rightarrow\left(\right.$ в). Если $\widehat{x}_{i} \| \widehat{x}_{\pi(i)}^{\prime}$ для некоторой перестановки $\pi$, то $\widetilde{X}_{i}=\widetilde{X}_{\pi(i)}^{\prime}, i=$ $\{1,2\}$. Поэтому $B_{0}(\omega)=\widetilde{X}_{1} \cap \widetilde{X}_{2}=\widetilde{X}_{1}^{\prime} \cap \widetilde{X}_{2}^{\prime}=B_{0}\left(\omega^{\prime}\right), B_{1}(\omega)=\widetilde{X}_{1} \cup \widetilde{X}_{2}=\widetilde{X}_{1}^{\prime} \cup$ $\widetilde{X}_{2}^{\prime}=B_{1}\left(\omega^{\prime}\right)$.

\section{§4. Поливекторы ранга 2 над полем. "2-плюккеровых" колец не существует}

В этом параграфе полностью решается вопрос о необходимых и достаточных условиях 2-разложимости произвольного $m$-вектора $\omega \in \Lambda^{m}\left(A^{n}\right)$, где $A$ - поле, и доказывается, что единственными кольцами, в которых $\operatorname{rank}_{F r} \omega=\operatorname{rank} \omega$, являются поля.

Теорема 4.1. Пусть А-коммутативное кольцо. Следующие условия эквивалентны:

(а) кольцо А является полем;

(б) необходимым и достаточным условием 2-разложимости произвольного т-вектора $\omega \in \Lambda^{m}\left(A^{n}\right)$ является равенство $b_{0}(\omega)+b_{1}(\omega)=2 m$;

(в) $\operatorname{rank} \omega=\operatorname{rank}_{\mathrm{Fr}} \omega$ для любого т-вектора $\omega \in \Lambda^{m}\left(A^{n}\right)$, у которого $\operatorname{rank}_{\mathrm{Fr}} \omega=2$

(г) $\operatorname{rank} \omega=\operatorname{rank}_{\mathrm{Fr}} \omega \partial л я$ любого m-вектора $\omega \in \Lambda^{m}\left(A^{n}\right)$.

ДокАЗАТЕльство. (а) $\Rightarrow(б)$. Ввиду леммы 2.1 2-разложимость поливектора $\omega$ равносильна 2-разложимости фигурируюшего в этой лемме поливектора $\omega^{\prime}$, а равенство $b_{0}(\omega)+b_{1}(\omega)=2 m$ равносильно равенству $b_{1}\left(\omega^{\prime}\right)=2\left(m-b_{0}(\omega)\right)$. Таким образом, достаточно доказать утверждение для поливектора $\omega^{\prime}$, т.е. ограничиться рассмотрением таких векторов $\omega$, что $b_{0}(\omega)=0$.

Если такой поливектор 2-разложим, то он представим в виде $\omega=\widehat{x}+\widehat{y}$, где $\operatorname{Supp} \widehat{x} \cap \operatorname{Supp} \widehat{x}=0$. Значит, $b_{1}(\omega)=\operatorname{dim} \operatorname{Supp} \omega=2 m$.

Докажем обратное утверждение. Более того, мы докажем, что если $b_{1}(\omega) \geqslant$ $2 m$, то $\operatorname{rank} \omega \leqslant 2$.

По условию в пространстве $B_{1}(\omega)$ есть $2 m$ линейно независимых векторов $e_{1}, \ldots, e_{2 m}$. Дополним эту систему до базиса $e_{1}, \ldots, e_{n}$ пространства $A^{n}$ и разложим $\omega$ по этому базису. Пусть $\omega=e_{1} \wedge u+v$, где в разложении поливекторов $u, v$ нет $e_{1}$. Так как поливектор $\omega \wedge e_{1}=v \wedge e_{1}$ разложим, то и $v$ разложим, т.е. $v=\widehat{y}$. 
По крайней мере $m-1$ векторов из $e_{2}, \ldots, e_{2 m}$ линейно независимы по модулю $\left\{e_{1}, y_{1}, \ldots, y_{m}\right\}$. Пусть таковы $e_{2}, \ldots, e_{m}$. Заменим базис пространства $A^{n}$, оставив неизменными первые $m$ базисных векторов $e_{1}, \ldots, e_{m}$, положив $e_{m+1}=y_{1}, \ldots$, $e_{2 m}=y_{m}$ и выбрав остальные базисные векторы произвольным образом. Пусть в новом базисе $u=e_{j} \wedge u^{\prime}+u^{\prime \prime}$, где $2 \leqslant j \leqslant m$, и в разложение поливекторов $u^{\prime}, u^{\prime \prime}$ не входит $e_{j}$.

Так как поливектор $\omega \wedge e_{j}=e_{1} \wedge u^{\prime \prime} \wedge e_{j}+\widehat{y} \wedge e_{j}$ разложим, то и поливектор $q=$ $e_{1} \wedge u^{\prime \prime}+\widehat{y}$ разложим, т.е. для его координат выполняются соотношения Плюккера вида (3), в том числе для всякого $2 \leqslant k \leqslant m$ вьполняются все соотношения

$$
a_{i_{1}, \ldots, i_{m-1}, k} \cdot a_{m+1, \ldots, 2 m}+\sum_{s=1}^{m} a_{i_{1}, \ldots, i_{m-1}, m+s} \cdot a_{k, m+1, \ldots, \widehat{m+s}, \ldots, 2 m}=0,
$$

где $a_{i_{1}, \ldots, i_{m}}-$ коэффициент в разложении $q$ при $e_{i_{1}} \wedge \cdots \wedge e_{i_{m}}, i_{1}<\cdots<i_{m}$. Здесь во втором слагаемом все вторые сомножители нулевые (так как это коэффициенты при мономах, не зависящих от $e_{1}$ и не пропорциональных $\left.\widehat{y}\right)$. Кроме того, коэффициент при мономе $\widehat{y}$ единичен, $a_{m+1, \ldots, 2 m}=1$. Следовательно, соотношение вырождается в равенство

$$
a_{i_{1}, \ldots, i_{m-1}, k}=0
$$

для любого набора $i_{1}, \ldots, i_{m-1}$.

Таким образом, для всякого $2 \leqslant j \leqslant m$ мы получаем $u^{\prime \prime}=0$, т.е. $(m-1)$-вектор $u$ делится на каждый из $m-1$ векторов $e_{2}, \ldots, e_{m}$. Значит, $e_{1} \wedge u=\alpha e_{1} \wedge \cdots \wedge e_{m}$, $\alpha \in A$, и поливектор $\omega=e_{1} \wedge u+\widehat{y}$ 2-разложим.

$(б) \Rightarrow(в)$. Пусть $\omega-m$-вектор и $\operatorname{rank}_{\mathrm{Fr}} \omega=2$. Тогда из (б) следует, что $\operatorname{dim} B_{0}^{\mathrm{Fr}}(\omega)+\operatorname{dim} B_{1}^{\mathrm{Fr}}(\omega)=2 m$. Но $B_{i}(\omega)=B_{i}^{\mathrm{Fr}}(\omega) \cap A^{n}$, поэтому $b_{i}(\omega)=$ $\operatorname{rank} B_{i}(\omega)=\operatorname{dim} B_{i}^{\mathrm{Fr}}(\omega), i=1,2$, так что $b_{1}(\omega)+b_{0}(\omega)=2 m$. По условию (б) этого достаточно для 2-разложимости $\omega$. Следовательно, $\operatorname{rank} \omega=\operatorname{rank}_{\mathrm{Fr}} \omega=2$.

$($ в $\Rightarrow(\mathrm{a})$. Предположим, что в $A$ сушествует ненулевой необратимый элемент $a$. Рассмотрим следующий 3-вектор $\omega \in \Lambda^{3}\left(A^{6}\right)$ :

$$
\begin{aligned}
\omega= & e_{1} \wedge e_{2} \wedge e_{3}+e_{1} \wedge e_{2} \wedge e_{6}+a e_{1} \wedge e_{5} \wedge e_{6}-e_{1} \wedge e_{3} \wedge e_{5} \\
& +e_{2} \wedge e_{3} \wedge e_{4}-a e_{2} \wedge e_{4} \wedge e_{6}+a e_{3} \wedge e_{4} \wedge e_{5}+a^{2} e_{4} \wedge e_{5} \wedge e_{6} .
\end{aligned}
$$

Покажем, что rank $\mathrm{Fr}_{\mathrm{F}} \omega=2$. Пусть $x_{1}=a^{-1} e_{1}+e_{4}, x_{2}=e_{2}+a e_{5}, x_{3}=e_{3}+a e_{6}$, $x_{4}=\left(1-a^{-1}\right) e_{1}, x_{5}=e_{2}, x_{6}=e_{3}$. Тогда $x_{1} \wedge x_{2} \wedge x_{3}+x_{4} \wedge x_{5} \wedge x_{6}=\omega$, где $x_{i} \in \mathrm{Fr}^{6}$, значит, $\operatorname{rank}_{\mathrm{Fr}} \omega \leqslant 2$. Так как

$$
x_{1} \wedge x_{2} \wedge x_{3} \wedge x_{4} \wedge x_{5} \wedge x_{6}=-a^{2}\left(1-a^{-1}\right) e_{1} \wedge e_{2} \wedge e_{3} \wedge e_{4} \wedge e_{5} \wedge e_{6} \neq 0
$$

то $B_{0}(\omega)=0$. Следовательно, $\operatorname{rank}_{\mathrm{Fr}} \omega=2$. Отсюда по условию $\operatorname{rank} \omega=2$, т.е. $\omega=y_{1} \wedge y_{2} \wedge y_{3}+y_{4} \wedge y_{5} \wedge y_{6}$, где $y_{i} \in A^{6}$. Поскольку $\omega$ не является произведением 2-вектора на разложимый поливектор, можно воспользоваться теоремой единственности 3.1, согласно которой $x_{1} \wedge x_{2} \wedge x_{3}=y_{1} \wedge y_{2} \wedge y_{3}$ (может быть, после перенумеровки $\left.y_{1}, \ldots, y_{6}\right)$. Отсюда следует, что $a^{-1} e_{1} \wedge e_{2} \wedge e_{3} \in \Lambda^{3}\left(A^{6}\right)$, что означает обратимость элемента $a \in A$.

$($ г) $\Rightarrow($ в). Очевидно.

(a) $\Rightarrow($ г). Очевидно, так как если $A-$ поле, то $A=\operatorname{Fr}(A)$. 
СлеДСтвиЕ 4.1. Пусть $A$ - поле, $\omega \in \Lambda^{m}\left(A^{n}\right)$ - ненулевой m-вектор, причем $b_{0}(\omega)<m-2$. Для того чтобъ $\operatorname{rank} \omega$ бъл равен 2 , необходимо и достаточно, чтобъ $B_{1}(\omega)=M_{1} \cup M_{2}, B_{0}(\omega)=M_{1} \cap M_{2}$, где $M_{1}, M_{2}$ m-мерные подпространства в $A^{n}$. Если әти условия выполнены, то $\omega=$ $\widehat{x}_{1}+\widehat{x}_{2}$, дде $\widehat{x}_{i} \in \Lambda^{m}\left(M_{i}\right), i=1,2$.

ДокАЗАТЕЛЬСТво. Если $\omega=\widehat{x}_{1}+\widehat{x}_{2}$, где $\widehat{x}_{1}, \widehat{x}_{2}-$ разложимые $m$-векторы, то ввиду предложения $2.1 B_{1}(\omega)=X_{1} \cup X_{2}$, а в силу леммы $2.1 B_{0}(\omega)=X_{1} \cap X_{2}$. Обратно, если $B_{1}(\omega)=M_{1} \cup M_{2}, B_{0}(\omega)=M_{1} \cap M_{2}$, где $M_{1}, M_{2}-m$-мерные подпространства в $A^{n}$, то

$$
\operatorname{dim} E_{1}(\omega)=\operatorname{dim}\left(M_{1}+M_{2}\right)=2 m-\operatorname{dim}\left(M_{1} \cap M_{2}\right)=2 m-\operatorname{dim} B_{0}(\omega) .
$$

Достаточность теперь вытекает из теоремы 4.1.

Пусть $\omega=\widehat{x}_{1}+\widehat{x}_{2}$. Рассмотрим $m$-вектор $\omega^{\prime}=\widehat{x}_{1}^{\prime}+\widehat{x}_{2}^{\prime}$, где $\widehat{x}_{i} \in \Lambda^{m}\left(M_{i}\right)$. Так как $B_{1}\left(\omega^{\prime}\right)=X_{1}^{\prime} \cup X_{2}^{\prime}$ (предложение 2.1), $B_{0}\left(\omega^{\prime}\right)=X_{1}^{\prime} \cap X_{2}^{\prime}$ (лемма 2.1), где $X_{i}^{\prime}=B_{0}\left(\widehat{x}_{i}^{\prime}\right)=M_{i}, i=1,2$, то $B_{0}\left(\omega^{\prime}\right)=B_{0}(\omega), B_{1}\left(\omega^{\prime}\right)=B_{1}(\omega)$, откуда в силу предложения $3.1 \widehat{x}_{i} \| \widehat{x}_{i}^{\prime}$. Таким образом, $\widehat{x}_{i}^{\prime} \in \Lambda^{m}\left(M_{i}\right), i=1,2$.

Как видно из доказанной теоремы, если $A \neq \operatorname{Fr}(A)$, то необходимое условие $\operatorname{rank}_{F r} \omega=2$ не является, вообще говоря, достаточным для 2-разложимости $\omega$. Однако для 2-векторов над нётеровыми плюккеровыми кольцами это так [10]. Аналогичньй результат имеет место и для $m$-векторов, являющихся произведением 2-вектора на разложимый.

ПРЕДЛОЖЕНИЕ 4.1. Пусть $A$ - кольцо главных идеалов, $\omega-m$-вектор, для которого $b_{0}(\omega)=m-2$. Тогда $\operatorname{rank} \omega=2$ в том и только том случае, когда $\operatorname{rank}_{\mathrm{Fr}} \omega=2$.

Лемма 4.1. Пусть $A$ - плюккерово кольцо, $\omega \in \Lambda^{m}\left(A^{n}\right), x \in A^{n+p}, p>0$, $x \notin A^{n}$. Тогдa $\operatorname{rank}(\omega \wedge x)=\operatorname{rank} \omega$.

ДокАЗАТЕЛЬСТво. Очевидно, $\operatorname{rank}(\omega \wedge x) \leqslant \operatorname{rank} \omega$. Пусть

$$
\omega \wedge x=x_{1}^{1} \wedge \cdots \wedge x_{k}^{1}+\cdots+x_{1}^{l} \wedge \cdots \wedge x_{k}^{l}
$$

- минимальное разложение $(m+1)$-вектора $\omega \wedge x \in \Lambda^{m}\left(A^{n} \oplus A x\right)$. Пусть $x_{j}^{i}=y_{j}^{i}+$ $a_{j}^{i} x$, где $y_{j}^{i} \in A^{n}, a_{j}^{i} \in A, i=1, \ldots, l, j=1, \ldots, m+1$. Тогда

$$
\widehat{x}_{i}=x_{1}^{i} \wedge \cdots \wedge x_{m+1}^{i}=y_{1}^{i} \wedge \cdots \wedge y_{m+1}^{i}+x \wedge \mu_{i},
$$

где $\mu_{i}-m$-вектор из $\Lambda^{m}\left(A y_{1}^{i} \oplus \cdots \oplus A y_{m+1}^{i}\right), i=1, \ldots, l$. Все $m$-векторы над свободньм модулем ранга $(m+1)$ в ОР-кольце разложимы [8], поэтому $\mu_{i}=\widehat{\mu}_{i}$, $i=1, \ldots, l$. Теперь $\omega \wedge x=y_{1}^{1} \wedge \cdots \wedge y_{m+1}^{1}+\cdots+y_{1}^{l} \wedge \cdots \wedge y_{m+1}^{l}+x \wedge \sum_{i=1}^{l} \widehat{\mu}_{i}$. Отсюда $\left(\omega \pm \sum_{i=1}^{l} \widehat{\mu}_{i}\right) \wedge x=\widehat{y}_{1}+\cdots+\widehat{y}_{l} \in \Lambda^{m}\left(A^{n}\right)$, что возможно лишш в случае $\omega= \pm \sum_{i=1}^{l} \widehat{\mu}_{i}$. Значит, $\operatorname{rank}(\omega \wedge x) \geqslant \operatorname{rank} \omega$. 
ДоКАЗАТЕЛЬСТВО ПРЕДЛОЖЕНИЯ 4.1. Так как $B_{0}(\omega)$ - замкнутый подмодуль в $A^{n}$, то сушествует базис $e_{1}, \ldots, e_{n}$ модуля $A^{n}$, первые $m-2$ вектора которого являются базисом для $B_{0}(\omega)$. Пусть

$$
\omega=\sum_{1 \leqslant i_{1}<\cdots<i_{m} \leqslant n} e_{i_{1}} \wedge \cdots \wedge e_{i_{m}}
$$

И

$$
\omega^{\prime}=\sum_{m-1 \leqslant i_{1}<i_{2} \leqslant n} a_{1, \ldots, m-2, i_{1}, i_{2}} e_{i_{1}} \wedge e_{i_{2}} .
$$

Нетрудно проверить, что

$$
\omega=e_{1} \wedge \cdots \wedge e_{m-2} \wedge \omega^{\prime}
$$

Так как $\omega^{\prime} \in \Lambda^{m}\left(A e_{m-1} \oplus \cdots \oplus A e_{n}\right)$, то ввиду леммы 4.1

$$
\operatorname{rank} \omega=\operatorname{rank}\left(e_{1} \wedge \cdots \wedge e_{m-2} \wedge \omega^{\prime}\right)=\operatorname{rank} \omega^{\prime}
$$

Воспользовавшись теоремой 2 из [10] о ранге 2-векторов над нётеровым плюккеровьм кольцом, получаем требуемьй результат.

Множество всех поливекторов над данным кольцом можно разбить на следующие четыре класса:

1) 2-векторы;
2) $m$-векторы с $m>2$ и $b_{0}=m-2$;
3) $m$-векторы с $m>2$ и $b_{0}>m-2$;
4) $m$-векторы с $m>2$ и $b_{0}<m-2$.

Как видно из теоремы 2 из [10] и предложения 4.1, в случае плюккеровых колец вопрос о 2-разложимости поливекторов первых трех классов сводится в принципе к вопросу об их 2-разложимости над полем частных. Иначе обстоит дело с $m$-векторами $(m>2)$, у которых $b_{0}<m-2$. Исследованию условий 2-разложимости таких $m$-векторов посвящен $\S 5$.

\section{§5. Поливекторы ранга 2 над кольцом Крулля. Общий случай}

Основными результатами этого параграфа являются предложение 5.1, содержащее достаточные условия 2-разложимости $m$-вектора $\omega$ с $b_{0}<m-2$, и теорема 5.2, в которой установлены необходимые и достаточные условия 2-разложимости для так называемых 2-примитивных $m$-векторов.

Лемма 5.1. Пусть $A-$ кольцо Крулля, $M_{1}, M_{2}$ - замкнутье подмодули свободного модуля $A^{n}$ ранга $m$, причем ни один из них не лежст в другом. Если $M_{1}+M_{2}$ замкнут в $A^{n}$, то $\Lambda^{m}\left(M_{1}\right)+\Lambda^{m}\left(M_{2}\right)=\widehat{\Lambda^{m}\left(M_{1}\right)}+\widehat{\Lambda^{m}\left(M_{2}\right)}$. 
ДоказАтельство. Очевидно, $\left.\widehat{\Lambda^{m}\left(M_{i}\right.}\right) \subset \Lambda^{m}\left(M_{1} \widetilde{)^{+} \Lambda^{m}}\left(M_{2}\right)\right.$. Покажем, что $\Lambda^{m}\left(M_{1} \widehat{)+\Lambda^{m}}\left(M_{2}\right) \subset \widehat{\Lambda^{m}\left(M_{1}\right)}+\widehat{\Lambda^{m}\left(M_{2}\right.}\right)$. Пусть $\omega \in \Lambda^{m}\left(M_{1}\right)+\Lambda^{m}\left(M_{2}\right)$, т.е. $a \omega=\widehat{x}_{1}+\widehat{x}_{2}$, где $\widehat{x}_{i} \in \Lambda^{m}\left(M_{i}\right), i=1,2$. Покажем, что $\widehat{x}_{i} / a \in \Lambda^{m}\left(A^{n}\right)$. Для этого достаточно доказать, что $\operatorname{div} \widehat{x}_{i}$ делится на $a$, причем можно ограничиться доказательством в локализации по простому идеалу $p$ высоты 1 . Будем считать, что вместо $A$ рассматривается $A_{p}$ и все модули локализованы по $p$. Так как $M_{1}$ и $M_{2}$ замкнуты, $M_{1} \cap M_{2}$ тоже замкнут [8] и, следовательно, выделяется в $M_{2}$ прямым слагаемым. Пусть $M_{2}=N \oplus\left(M_{1} \cap M_{2}\right)$. Тогда $M_{1}+M_{2}=M_{1} \oplus N$. Пусть $e_{1}, \ldots, e_{m}$ - базис в $M_{1}$ и $f_{1}, \ldots, f_{t}-$ базис в $N$. Тогда $\operatorname{div}\left(e_{1} \wedge \cdots \wedge e_{m} \wedge\right.$ $\left.f_{1} \wedge \cdots \wedge f_{t}\right)=1$. Очевидно, $\widehat{x}_{1}=a_{1} \widehat{e}\left(a_{1} \in A\right)$, а $f_{1} \wedge \cdots \wedge f_{t} \wedge \widehat{x}_{2}=0$, так как $f_{i} \in N \subset M_{2}$ и $f_{1} \wedge \cdots \wedge f_{t} \wedge \widehat{x}_{2} \in \Lambda^{m+t}\left(M_{2}\right)=0$. Следовательно, $a \omega \wedge \widehat{f}=\widehat{x}_{1} \wedge \widehat{f}=$ $a_{1} \widehat{e} \wedge \widehat{f}$. Переходя к дивизорам, получаем, что $a \operatorname{div}(\omega \wedge \widehat{f})=a_{1} \operatorname{div}(\widehat{e} \wedge \widehat{f})=1$. Следовательно, локально $\operatorname{div} \widehat{x}_{1}=a_{1}$ делится на $a$. Лемма доказана.

Пусть $M$ - замкнутый подмодуль ранга $m$ в свободном модуле $A^{n}$. Назовем модуль $M$ плюккеровым, если всякий $m$-вектор $\omega$, для которого $B_{0}(\omega)=M$, разложим. Кольцо $A$ тогда и только тогда плюккерово, когда все замкнутые модули над ним плюккеровы.

ПрЕДЛОЖЕНИЕ 5.1. Пусть $A-$ кольио Крулля, $\omega \in \Lambda^{m}\left(A^{n}\right)$, причем $b_{0} \neq$ m-2. Предположсм, что выполнены условия:

(а) $B_{1}(\omega)=M_{1} \cup M_{2}, B_{0}(\omega)=M_{1} \cap M_{2}$, где $M_{1} \neq M_{2}-$ плюккеровьи модули ранга $m$;

(б) $E_{1}(\omega)-$ замкнутый модуль.

Тогда $\operatorname{rank} \omega=2$.

ДокаЗАтельство. Рассмотрим $\omega$ как элемент $\Lambda^{m}\left(\mathrm{Fr}^{n}\right)$. Как уже отмечалось, $b_{1}(\omega)=\operatorname{dim} B_{1}^{\mathrm{Fr}}(\omega)$. Если $b_{0}(\omega)=m$, то $B_{0}(\omega) \subset M_{1}$ - замкнутые модули ранга $m$, поэтому $B_{0}(\omega)=M_{1} \cap M_{2}=M_{1}=M_{2}$, что противоречит условию. Следовательно, $b_{0}(\omega)<m-2$. Ввиду следствия $4.1 \omega=\widehat{x}_{1}+\widehat{x}_{2}$, где $\widehat{x}_{i} \in \Lambda^{m}\left(\operatorname{Fr} M_{i}\right)$. Отсюда $\omega \in \Lambda^{m}\left(M_{1} \widehat{+\Lambda^{m}}\left(M_{2}\right)\right.$. Так как ранги замкнутых модулей $M_{1}$ и $M_{2}$ равны, то ни один из них не лежит в другом. Кроме того, очевидно, $E_{1}(\omega)=M_{1}+M_{2}$, значит, можно применить лемму 5.1. Таким образом, $\omega \in \widehat{\Lambda^{m\left(M_{1}\right)}}+\widehat{\left.\Lambda^{m\left(M_{2}\right.}\right)}$, т.е. $\omega=\widehat{y}_{1} / a+\widehat{y}_{2} / a$, где $\widehat{y}_{i} \in \Lambda^{m}\left(M_{i}\right), \widehat{y}_{i} / a \in \Lambda^{m}\left(A^{n}\right), i=1,2$. Так как $M_{i}$ - плюккеровы $A$-модули, то $\widehat{y}_{i} / a=\widehat{z}_{i} \in \Lambda^{m}\left(M_{i}\right), i=1,2$. Следовательно, $\operatorname{rank} \omega=2$.

ЗАмЕчАниЕ. Условие (а) является также и необходимым (см. следствие 4.1). Условие (б) в обшем случае необходимо тогда и только тогда, когда $A$ - поле.

Tеорема 5.1. Пусть $A-$ кольио Крулля, $\omega \in \Lambda^{m}\left(A^{n}\right)$, причем $b_{0} \neq m-2$. Если $B_{1}(\omega)=F_{1} \cup F_{2}, B_{0}(\omega)=F_{1} \cap F_{2}$, где $F_{1} \neq F_{2}-$ свободные модули ранга $m$, а $E_{1}(\omega)$ - замкнутый модуль, то $\operatorname{rank} \omega=2$.

ДоКАЗАТЕЛЬСТво. Для того чтобы применить предложение 5.1, достаточно доказать, что модули $F_{1}, F_{2}$ плюккеровы. Очевидно, $B_{1}(\omega)$ - замкнутое подмножество в $A^{n}$, так как если $x \wedge \omega$-плюккеров $m$-вектор и $x=a y$, где $y \in A^{n}$, то плюккеровым будет и $(m+1)$-вектор $y \wedge \omega \in \Lambda^{m+1}\left(A^{n}\right)$. Согласно [8; следствие 
из предложения 1.1] наибольший общий дивизор всех элементов $\Lambda^{m}\left(F_{i}\right)$ равен 1. Так как $\Lambda^{m}\left(F_{i}\right)=A f_{1}^{i} \wedge \cdots \wedge f_{m}^{i}$, где $f_{1}^{i}, \ldots, f_{m}^{i}$ - образующие модуля $F_{i}$, то $\operatorname{div}\left(f_{1}^{i} \wedge \cdots \wedge f_{m}^{i}\right)=1, i=1,2$. Отсюда можно вывести, что $\Lambda^{m}\left(F_{i}\right)$ - замкнутый модуль. Следовательно, $\widehat{\Lambda^{m}\left(F_{i}\right)}=\Lambda^{m}\left(F_{i}\right)$. Таким образом, $F_{1}, F_{2}$-плюккеровы модули. Теперь утверждение теоремы вытекает из предложения 5.1.

СлеДСтвИЕ 5.1. Пусть $A-$ плюккерово кольио Крулля, $\omega \in \Lambda^{m}\left(A^{n}\right)$, причем $b_{0}(\omega)<m-2 u \operatorname{rank}_{\mathrm{Fr}} \omega=2$. Если модуль $E_{1}(\omega)$ замкнут, то $\operatorname{rank} \omega=2$.

Перейдем теперь к формулировке и доказательству критерия 2-разложимости для примитивных $m$-векторов над кольцом Крулля. Соответствующая теорема для 1-разложимых $m$-векторов доказана в [9].

Обращение к классу примитивных поливекторов при исследовании вопросов разложимости объясняется следующими причинами. Как известно, если $A$-поле, то условия разложимости $\omega \in \Lambda^{m}\left(A^{n}\right)$ выражаются в виде ограничений на размерность пространства $B_{0}(\omega)$. В случае произвольного коммутативного кольца условие разложимости $m$-вектора $\omega$, сформулированные в терминах свойств замкнутого модуля $B_{0}(\omega)$, обеспечивают разложимость всех $m$-векторов $\omega^{\prime} \in \Lambda^{m}\left(A^{n}\right)$, параллельных $m$-вектору $\omega$. Если модуль $B_{0}(\omega)$ свободен или кольцо $A$ факториально, то множество таких $\omega^{\prime}$ имеет вид $A \omega_{0}$, где $\omega_{0}$-поливектор с единичным дивизором, у которого $B_{0}\left(\omega_{0}\right)=B_{0}(\omega)$. Так как из разложимости $\omega_{0}$ следует разложимость всех $\omega \in \Lambda^{m}\left(A^{n}\right)$, для которых $B_{0}(\omega)=B_{0}\left(\omega_{0}\right)$, то вопрос о разложении $\omega$ сводится, таким образом, к вопросу о разложимости примитивного поливектоpa $\omega_{0}$.

В случае 2-разложимости можно ввести понятие примитивности таким образом, чтобы описанная ситуация в основном сохранилась. Условие примитивности (или, как мы будем в дальнейшем его назьвать, 1-примитивности) эквивалентно в кольце Крулля тому, что для любого простого дивизора $p \bar{\omega} \neq 0$ (через $\bar{\omega}$ обозначен образ $\omega$ в факторкольце $A / p)$. Иньми словами, $\omega$ 1-примитивен тогда и только тогда, когда не существует такого простого дивизора $p$, что $\operatorname{rank}_{\mathrm{Fr}} \bar{\omega}=\operatorname{rank} \bar{\omega}=0$.

Так как $\operatorname{rank} \omega=1$ в том и только том случае, когда для координат $\omega$ выполнены соотношения Плюккера (3) $\Pi_{\omega}\left(i_{1}, \ldots, i_{m-1} \mid j_{0}, \ldots, j_{m}\right)=0$, то для 2-примитивности $\omega$ необходимо и достаточно, чтобы дивизор идеала, порожденного левьми частями соотношений Плюккера, был равен 1. Это дает основание обозначить $\operatorname{div}_{1 \leqslant i_{1} \leqslant \cdots \leqslant i_{m-1} \leqslant j_{0} \leqslant \cdots \leqslant j_{m} \leqslant n}\left\{\Pi_{\omega}\left(i_{1}, \ldots, i_{m-1} \mid j_{0}, \ldots, j_{m}\right)\right\}$ через $\operatorname{div}_{2} \omega$; $m$-вектор $\omega 2$-примитивен тогда и только тогда, когда $\operatorname{div}_{2} \omega=1$. Очевидно, $\operatorname{div} \omega=\operatorname{div}_{1} \omega$ делит $\operatorname{div}_{2} \omega$, поэтому 2 -примитивный $m$-вектор является 1-примитивным.

Пусть, далее, $p$ - некоторый простой дивизор. Рассмотрим факторкольцо $\bar{A}=A / p$, отображения $\varphi_{p}: A \rightarrow \bar{A}$ и $\varphi_{p}^{n}: A^{n} \rightarrow \bar{A}^{n}$. Пусть $M$ - подмножество в $A^{n}, \bar{M}$ - его образ при отображении $\varphi_{p}^{n}$ и $r(\bar{M})$ - ранг множества $\bar{M}$ относительно кольца $\bar{A}$, т.е. наибольшее число линейно независимых над $\bar{A}$ векторов из $\bar{M}$. Обозначим через $r_{0}(M)$ наименьшее из чисел $r\left(\varphi_{p}^{n} M\right)$ для различных простых дивизоров $p$.

В [8] были введены инварианты $d_{i}(M)$ подмодуля $M$ свободного модуля - аналог элементарных делителей. По определению $d_{i}(M)=\operatorname{div} \Lambda^{i}(M), i=1, \ldots, m=$ 
$\operatorname{rank} M$. Покажем, что $r_{0}(M)=\max \left\{s \in \mathbb{Z}: d_{s}(M)=1\right\}$. В самом деле, если $d_{s}(M)=1$, то каков бы ни был простой дивизор $p$, существует разложимый элемент $x_{1} \wedge \cdots \wedge x_{s} \in \Lambda^{s}(M)$, координаты которого не все кратны $p$. Следовательно, для любого дивизора $p$ сушествуют линейно независимые элементы $\bar{x}_{1}, \ldots, \bar{x}_{s} \in \bar{M}$. Отсюда $r(\bar{M}) \geqslant s$ и $r_{0}(M) \geqslant \max \left\{s \in \mathbb{Z}: d_{s}(M)=1\right\}$. Если $d_{s}(M) \neq 1$, то сушествует простой дивизор $p$, делящий все элементы $\Lambda^{s}(M)$. Следовательно, $r_{0}(M)=\max \left\{s \in \mathbb{Z}: d_{s}(M)=1\right\}$.

Заметим, что если $M$ - замкнутый модуль, то $d_{m}(M)=1$, так что $r_{0}(M)=$ $\operatorname{rank} M$. В случае, когда $M$ рефлексивен, согласно [8; следствие 1] верно и обратное: если $r_{0}(M)=\operatorname{rank} M$, то $M$ - замкнутый модуль. Таким образом, разность $\operatorname{rank} M-r_{0}(M)$ определяет меру замкнутости модуля $M$.

ТеОРема 5.2. Пусть $A-$ кольцо Крулля, $\omega$ - 2-примитивный т-вектор над $A^{n}$ с $b_{0}(\omega)<m-2$. Следующие условия әквивалентны:

(a) $\operatorname{rank} \omega=2$;

(б) ранг любого т-вектора $\omega^{\prime} \in \Lambda^{m}\left(A^{n}\right)$ с $B_{i}\left(\omega^{\prime}\right)=B_{i}(\omega), i=1,2$, равен 2 ;

(в) $B_{1}(\omega)=F_{1} \cup F_{2}, B_{0}(\omega)=F_{1} \cap F_{2}$, где $F_{1} \neq F_{2}$ - свободные подмодули ранга $т$ в $A^{n}$, для которых $\Lambda^{m}\left(F_{1}\right)+\Lambda^{m}\left(F_{2}\right)$ - замкнутый подмодуль $\Lambda^{m}\left(A^{n}\right)$;

(г) $B_{1}(\omega)=F_{1} \cup F_{2}, B_{0}(\omega)=F_{1} \cap F_{2}$, әде $F_{1} \neq F_{2}$ - свободнье подмодули ранга $m$ в $A^{n}$, и $r_{0}\left(E_{1}(\omega)\right) \neq m$.

ДокАЗАТЕльство. (а) $\Rightarrow\left(\right.$ в). Пусть $\omega=\widehat{x}_{1}+\widehat{x}_{2}$, где $\widehat{x}_{i}$ - разложимые $m$-векторы из $\Lambda^{m}\left(A^{n}\right), i=1,2$. Покажем, что $\operatorname{div} \widehat{x}_{i}=1, i=1,2$. В самом деле, предположим, что некоторый простой дивизор $p$ делит $\operatorname{div} \widehat{x}_{1}$. Тогда $\omega \equiv \widehat{x}_{2}$ по модулю $p$, т.е. $\bar{\omega}$ - плюккеров $m$-вектор над $\bar{A}^{n}$. Следовательно, его координаты удовлетворяют соотношениям Плюккера по модулю $p$, а дивизор идеала, порожденного левыми частями соотношений Плюккера, $\operatorname{div} \Pi_{\omega}=\operatorname{div}_{2} \omega$ кратен $p$. Это, однако, противоречит 2 -примитивности $\omega$, так что $\operatorname{div} \widehat{x}_{2}=1$. Таким образом, 2 -примитивньй $m$-вектор ранга 2 является суммой двух 1 -примитивных $m$-векторов $\widehat{x}_{1}, \widehat{x}_{2}$. В силу [9; теорема 1] отсюда следует, что $\widetilde{X}_{i}=X_{i}, i=1,2$. Ввиду следствия 2.1 и леммы $2.1 B_{1}(\omega)=X_{1} \cup X_{2}, B_{0}(\omega)=X_{1} \cap X_{2}$, где $X_{1} \neq X_{2}$ - свободные модули ранга $m$.

Докажем, далее, что $\Lambda^{m}\left(X_{1}\right)+\Lambda^{m}\left(X_{2}\right)$ - замкнутьй модуль. Пусть $a \mu=a_{1} \widehat{x}_{1}+$ $a_{2} \widehat{x}_{2}$, где $\mu \in \Lambda^{m}\left(A^{n}\right), a, a_{1}, a_{2} \in A$. Если $a_{2}$ не делится на $a$, то, умножив $a, a_{1}, a_{2}$ на некоторый элемент поля частных, можно считать, что сушествует простой дивизор $p$, делящий $a$ и не делящий $a_{2}$. По модулю этого дивизора $a_{1} \widehat{x}_{1} \equiv-a_{2} \widehat{x}_{2}$. Отсюда $a_{2} \omega \equiv\left(1-a_{1}\right) \widehat{x}_{1}$, причем $a_{2} \not \equiv 0$, что означает плюккеровость $m$-вектора $\bar{\omega} \in \Lambda^{m}\left(\bar{A}^{n}\right)$. Следовательно, координаты $\bar{\omega}$ удовлетворяют соотношениям Плюккера, так что $\operatorname{div} \Pi(\omega)$ кратен $p$. Это, однако, противоречит 2 -примитивности $\omega$, поэтому $a_{2}$ делится на $a$. По тем же причинам $a_{1}$ делится на $a$, значит, $\mu \in \Lambda^{m}\left(X_{1}\right)+$ $\Lambda^{m}\left(X_{2}\right)$.

$($ в $) \Rightarrow\left(\right.$ б). Пусть $\omega^{\prime} \in \Lambda^{m}\left(A^{n}\right)$ и $B_{1}\left(\omega^{\prime}\right)=F_{1} \cup F_{2}, B_{0}\left(\omega^{\prime}\right)=F_{1} \cap F_{2}$, где $F_{1}, F_{2}-$ свободные модули ранга $m$. Поскольку $B_{1}\left(\omega^{\prime}\right)$ - замкнутое множество, $F_{1}$ и $F_{2}$ замкнуты. Так как $\operatorname{dim} B_{i}^{\mathrm{Fr}}\left(\omega^{\prime}\right)=\operatorname{rank} B_{i}\left(\omega^{\prime}\right)$, то по следствию $4.1 \omega^{\prime}=\widehat{y}_{1}+\widehat{y}_{2}$, 
где $\widehat{y}_{i} \in \Lambda^{m}\left(\operatorname{Fr} F_{i}\right), i=1,2$. Это означает, что $\omega^{\prime} \in \Lambda^{m}\left(F_{1} \widetilde{++\Lambda^{m}}\left(F_{2}\right)\right.$. Поскольку $\Lambda^{m}\left(F_{1}\right)+\Lambda^{m}\left(F_{2}\right)$ замкнут, $\omega^{\prime} \in \Lambda^{m}\left(F_{1}\right)+\Lambda^{m}\left(F_{2}\right)$ и, следовательно, имеет ранг 2.

(б) $\Rightarrow($ а). Очевидно.

$\left(\right.$ в) $\Leftrightarrow(г)$. Так как $E_{1}(\omega)=F_{1}+F_{2}$, то для доказательства эквивалентности утверждений (в) и (г) достаточно проверить справедливость следуюшей леммы.

Лемма 5.2. Пусть $A$ - кольцо Крулля, $F_{1}, F_{2}$ - свободные замкнутые подмодули ранга $m$ в $A^{n}$. Подмодуль $\Lambda^{m}\left(F_{1}\right)+\Lambda^{m}\left(F_{2}\right)$ тогда и только тогда замкнут в $A^{n}$, когда $r_{0}\left(F_{1}+F_{2}\right) \neq m$.

ДокАЗАТЕЛЬСТво. Пусть $\Lambda^{m}\left(F_{1}\right)+\Lambda^{m}\left(F_{2}\right)$ замкнут. Если $r_{0}\left(F_{1}+F_{2}\right)=m$, то для некоторого простого дивизора $p \operatorname{rank} \varphi_{p}^{n}\left(F_{1}+F_{2}\right)=\operatorname{rank} \overline{\left(F_{1}+F_{2}\right)}=m$. Пусть $x_{1}^{i}, \ldots, x_{m}^{i}$ - образуюшие модуля $F_{i}, i=1,2, \widehat{x}_{i}=x_{1}^{i} \wedge \cdots \wedge x_{m}^{i}$. Очевидно, $\widehat{\bar{x}}_{i}=\bar{x}_{1}^{i} \wedge \cdots \wedge \bar{x}_{m}^{i} \in \Lambda^{m}\left(\bar{F}_{i}\right) \subset \Lambda^{m}\left(\overline{F_{1}+F_{2}}\right), \widehat{\bar{x}}_{i} \neq 0$, так как ввиду замкнутости модуля $F_{i} \operatorname{div} \overline{\bar{x}}_{i}=1$. Так как $\operatorname{rank} \Lambda^{m}\left(\overline{F_{1}+F_{2}}\right)$ равен 1 , то $\bar{a}_{1} \widehat{\bar{x}}_{1}=\bar{a}_{2} \widehat{\bar{x}}_{2}$ для некоторых ненулевых $\bar{a}_{1}, \bar{a}_{2} \in \bar{A}=A / p$. Возвращаясь в основное кольцо, получаем, что $a_{1} \widehat{x}_{1}=a_{2} \widehat{x}_{2}+\mu$, где $\mu-m$-вектор, все координаты которого делятся на $p$. В локализации по любому простому идеалу дивизор $p$ является главным, следовательно (поскольку $\Lambda^{m}\left(F_{1}\right)+\Lambda^{m}\left(F_{2}\right)$ замкнут также и локально), $a_{1}, a_{2}$ делятся на $p$. Делимость же, очевидно, сохраняется и в основном кольце, что противоречит требованию $\bar{a}_{1}, \bar{a}_{2}$.

Обратно, пусть $r_{0}\left(F_{1}+F_{2}\right) \neq m$. Предположим, что $\Lambda^{m}\left(F_{1}\right)+\Lambda^{m}\left(F_{2}\right)$ не замкнут. Тогда $a \mu=a_{1} \widehat{x}_{1}+a_{2} \widehat{x}_{2}$ для некоторого $m$-вектора $\mu$ и элементов $a, a_{1}, a_{2} \in A$ таких, что некоторый простой дивизор $p$, делящий $a$, не делит $a_{1}$ и $a_{2}$. Так как $\widehat{\bar{x}}_{i} \neq 0$, ранг $\overline{\left(F_{1}+F_{2}\right)}$ не может быть менше $m$. Умножив равенство $a \mu=a_{1} \widehat{x}_{1}+a_{2} \widehat{x}_{2}$ на $x_{j}^{2}$, получим, что $a_{1} \widehat{x}_{1} \wedge x_{j}^{2} \equiv 0(\bmod p), j=1, \ldots, m$, поэтому ранг модуля $\overline{\left(F_{1}+F_{2}\right)}$, порожденного векторами $\bar{x}_{1}^{1}, \ldots, \bar{x}_{m}^{1}, \bar{x}_{1}^{2}, \ldots, \bar{x}_{m}^{2}$, из которых лишш $m$ линейно независимы, равен $m$. Это противоречит предположению и доказывает замкнутость $\Lambda^{m}\left(F_{1}\right)+\Lambda^{m}\left(F_{2}\right)$. Доказательство теоремы закончено.

Теорема 5.1 дает легко проверяемые достаточные условия 2-разложимости поливекторов над кольцом Крулля. Последняя часть параграфа посвлщена выделению класса поливекторов ранга 2 , для которых эти условия необходимы.

Пусть $A$ - произвольное коммутативное кольцо. С каждым ненулевым $m$-вектором $\omega \in \Lambda^{m}\left(A^{n}\right)$ можно связать точную последовательность

$$
0 \longrightarrow B_{0}(\omega) \rightarrow A^{n} \stackrel{\psi_{\omega}}{\longrightarrow} A^{n} \wedge \omega \longrightarrow 0
$$

где $\psi_{\omega}(x)=x \wedge \omega$ для любого элемента $x \in A^{n}$. Очевидно, $\psi_{\omega}-$ линейное отображение, так что последовательность является точной последовательностью $A$-модулей и $A$-гомоморфизмов.

Фиксируем базис $e_{1}, \ldots, e_{n}$ модуля $A^{n}$. Пусть

$$
\omega=\sum_{1 \leqslant i_{1}<\cdots<i_{m} \leqslant n} a_{i_{1} \ldots i_{m}} e_{i_{1}} \wedge \cdots \wedge e_{i_{m}}
$$


где $a_{i_{1} \ldots i_{m}} \in A$. Так как $\psi_{\omega}: A^{n} \longrightarrow A^{n} \wedge \omega$ - линейное отображение, ему однозначно соответствует $\left(n \times C_{n}^{m+1}\right)$-матрица $Q_{\omega}$. Именно, поскольку $(m+1)$-векторы вида $e_{j_{1}} \wedge \cdots \wedge e_{j_{m+1}}\left(1 \leqslant j_{1}<\cdots<j_{m+1} \leqslant n\right)$ образуют базис в пространстве $\Lambda^{m+1}\left(A^{n}\right) \supset A^{n} \wedge \omega$, то $\psi_{\omega}\left(e_{i}\right)$ однозначно записывается в виде

$$
\psi_{\omega}\left(e_{i}\right)=\sum a_{i j_{1} \ldots j_{m+1}} e_{j_{1}} \wedge \cdots \wedge e_{j_{m+1}}
$$

где $a_{i j_{1} \ldots j_{m+1}}$ - элементы кольца $A$. Таким образом, $Q_{\omega}=\left(a_{i j_{1} \ldots j_{m+1}}\right)$. Элементы $a_{i j_{1} \ldots j_{m+1}}$ матрицы $Q_{\omega}$ следующим образом выражаются через координаты $a_{i_{1} \ldots i_{m}} m$-вектора $\omega$ :

$$
a_{i j_{1} \ldots j_{m+1}}= \begin{cases}(-1)^{\nu} a_{j_{1} \ldots \widehat{i} \ldots j_{m+1}}, & \text { если } i \in\left\{j_{1}, \ldots, j_{m+1}\right\}, \\ 0, & \text { если } i \notin\left\{j_{1}, \ldots, j_{m+1}\right\},\end{cases}
$$

где $\nu$ - четность перестановки $i j_{1} \ldots \widehat{i} \ldots j_{m+1}$.

Обозначим $i$-ю строку матрицы $Q_{\omega}$ через $Q_{\omega}^{i}$. Так как

$$
\psi_{\omega}\left(x_{1} e_{1}+\cdots+x_{n} e_{n}\right)=\sum_{i=1}^{n} x_{i} \sum_{1 \leqslant j_{1}<\cdots<j_{m+1} \leqslant n} a_{i j_{1} \ldots j_{m+1}} e_{j_{1}} \wedge \cdots \wedge e_{j_{m+1}}
$$

$A^{n} \wedge \omega$ изоморфен $A$-модулю, порожденному строками матрицы $Q_{\omega}$, а $B_{0}(\omega)$ изоморфен модулю, состоящему из таких векторов $x \in A^{n}$, что $x Q_{\omega}=x_{1} Q_{\omega}^{1}+\cdots+$ $x_{n} Q_{\omega}^{n}=0$. Так как $B_{0}(\omega)=\operatorname{ker} Q_{\omega}$, то, очевидно, $\operatorname{rank} B_{0}(\omega)+\operatorname{rank} Q_{\omega}=n$.

Назовем $m$-вектор $\omega \in \Lambda^{m}\left(A^{n}\right) \infty$-примитивньцм, если для любого элемента $a \in A \varphi_{a}^{n}\left(B_{0}(\omega)\right)=B_{0}\left(\Lambda^{m} \varphi_{a}^{n}(\omega)\right)$.

ПреДЛОЖЕНИЕ 5.2. Пусть $A$ - кольцо Крулля, $\omega \in \Lambda^{m}\left(A^{n}\right)-m$-вектор. Следующие условия әквивалентны:

(а) $\omega-\infty$-примитивный т-вектор;

(б) $A^{n} \wedge \omega-$ замкнутый подмодуль в $\Lambda^{m+1}\left(A^{n}\right)$;

(в) модуль $A^{n} \wedge \omega$ рефлексивен и $d_{r}\left(Q_{\omega}\right)=1$, где $r=\operatorname{rank} Q_{\omega}$.

ДокаЗАтЕльство. (а) $\Rightarrow\left(\right.$ б). Пусть $\mu \in \widetilde{A^{n} \wedge \omega}$, т.е. $\mu-(m+1)$-вектор, для которого сушествуют такие элементы $a_{0}, \ldots, a_{n} \in A$, что $a_{0} \mu=a_{1} Q_{\omega}^{1}+\cdots+a_{n} Q_{\omega}^{n}$.

Заметим, что $Q_{\Lambda^{m}} \varphi_{a_{0}}^{n}(\omega)=\left(\varphi_{a_{0}}^{n}\left(a_{i j_{1} \ldots j_{m+1}}\right)\right)$, поэтому в факторкольце по модулю $a_{0} \bar{a}_{1} Q \frac{1}{\omega}+\cdots+\bar{a}_{n} Q \frac{n}{\omega}=\bar{a}_{1} \bar{Q}_{\omega}^{1}+\cdots+\bar{a}_{n} \bar{Q}_{\omega}^{n}$. Следовательно, если через $а$ обозначить вектор $a=\left(a_{1}, \ldots, a_{n}\right)$, то $\bar{a} \bar{Q}_{\omega}=\bar{a} Q_{\bar{\omega}}=\overline{0}$, откуда $\bar{a} \in B_{0}(\bar{\omega})$. По условию $B_{0}(\bar{\omega})=\overline{B_{0}(\omega)}$, значит, $\bar{a}=\bar{x}$, где $x \in B_{0}(\omega)$. Таким образом, $a=x+a_{0} b$, где $b \in A^{n}$. Теперь $a_{0} b_{1} Q_{\omega}^{1}+\cdots+a_{0} b_{n} Q_{\omega}^{n}=\left(a_{1}-x_{1}\right) Q_{\omega}^{1}+\cdots+\left(a_{n}-x_{n}\right) Q_{\omega}^{n}=a_{1} Q_{\omega}^{1}+$ $\cdots+a_{n} Q_{\omega}^{n}=a_{0} \mu$, откуда $\mu=b Q_{\omega} \in A^{n} \wedge \omega$.

(б) $\Rightarrow\left(\right.$ a). Предположим, что $A^{n} \wedge \omega$ замкнут. Так как всегда $\overline{B_{0}(\omega)} \subset B_{0}(\bar{\omega})$, то достаточно доказать обратное включение. Пусть $x \in A^{n}, \bar{x} \in B_{0}(\bar{\omega})$, т.е. $\bar{x} \wedge \bar{\omega}=0$. Тогда $x \in \operatorname{ker} \psi_{\omega}=\operatorname{ker} \bar{\psi}_{\omega}$, откуда $\bar{x} \bar{Q}_{\omega}=0$. Следовательно, $x Q_{\omega}=a_{0} y$, где $y \in \Lambda^{m+1}\left(A^{n}\right), a \in A$. Таким образом, $y \in \widetilde{A^{n} \wedge \omega}=A^{n} \wedge \omega$. Это означает, 
что $y=\psi_{\omega}\left(x^{\prime}\right)$ для некоторого элемента $x^{\prime} \in A^{n}$. Отсюда $\left(a_{0} x^{\prime}-x\right) Q_{\omega}=0$, т.е. $a_{0} x^{\prime}-x \in B_{0}(\omega)$. Переходя к факторкольцу по модулю $a$, получаем, что $\bar{x} \in \overline{B_{0}(\omega)}$.

$(б) \Leftrightarrow($ в). Всякий замкнутый модуль $M$ ранга $r$ над кольцом Крулля рефлексивен и $d_{r}(M)=1[8]$. Обратно, если $M$ рефлексивен и $d_{r}(M)=1$, то он замкнут в силу [8; следствие предложения 1.1].

СлеДСТВИЕ 5.2. Пусть $A-$ кольцо Крулля, $\omega \in \Lambda^{m}\left(A^{n}\right), B_{0}(\omega)=0$. Тогда для $\infty$-примитивности $\omega$ необходимо и достаточно, чтобь $d_{r}(M)=1$.

Заметим, что условие 1 -примитивности $m$-вектора $\omega$ выражается в терминах матрицы $Q_{\omega}$ в виде $d_{1}(M)=1$. 2-примитивность означает, что для любого элемента $a \in A \bar{\omega}$ не является плюккеровым, т.е. rank $B_{0}(\bar{\omega})<m$. Но $B_{0}(\bar{\omega})=$ $\operatorname{ker} \bar{Q}_{\omega}$, поэтому $\operatorname{rank} B_{0}(\bar{\omega})<m$ тогда и только тогда, когда $\operatorname{rank} \bar{Q}_{\omega}>n-m$. В свою очередь, это эквивалентно условию $d_{n-m+1}\left(Q_{\omega}\right)=1$. Следовательно, $\omega$ 2 -примитивен тогда и только тогда, когда $d_{n-m+1}\left(Q_{\omega}\right)=1$. Теперь видно, что требование $\infty$-примитивности является самым сильньп из всех перечисленных условий примитивности.

ТеОРема 5.3. Пусть $A-$ кольио Крулля, $\omega \in \Lambda^{m}\left(A^{n}\right)-\infty$-примитивньи $m$-вектор, $b_{0}(\omega)<m-2$. Для того чтобь $\operatorname{rank} \omega=2$, необходимо и достаточно, чтобъ $E_{1}(\omega)$ бъл замкнут в $A^{n}$, а $B_{0}(\omega)$ и $B_{1}(\omega)$ представлялисв в виде $B_{1}(\omega)=F_{1} \cup F_{2}, B_{0}(\omega)=F_{1} \cap F_{2}$, әде $F_{1}, F_{2}$ - свободнье подмодули ранга $m$ в $A^{n}$.

ДоказАТЕльство. Достаточность вытекает из теоремы 5.1. Докажем необходимость. Так как $\infty$-примитивньй $m$-вектор является 2 -примитивным, то в силу теоремы 5.2 для $\infty$-примитивного $m$-вектора $\omega$ ранга 2 существуют такие свободные подмодули $F_{1}, F_{2} \subset A^{n}$, что $B_{1}(\omega)=F_{1} \cup F_{2}, B_{0}(\omega)=F_{1} \cap F_{2}$. Докажем, что $E_{1}(\omega)=F_{1}+F_{2}$ замкнут. Так как $B_{1}(\omega)$ замкнут, то $F_{1}, F_{2}$ - замкнутые подмодули.

ЛЕмма 5.3. Пусть $M_{1}$ и $M_{2}$ - замкнутье подмодули в $A^{n}$. Следующие условия әквивалентны:

(a) $M_{1}+M_{2}$ замкнут;

(б) для любого $a \in A \varphi_{a}^{n}\left(M_{1} \cap M_{2}\right)=\varphi_{a}^{n}\left(M_{1}\right) \cap \varphi_{a}^{n}\left(M_{2}\right)$;

ДоказАтельство. (а) $\Rightarrow\left(\right.$ б). Очевидно, $\varphi_{a}^{n}\left(M_{1} \cap M_{2}\right) \subset \varphi_{a}^{n}\left(M_{1}\right) \cap \varphi_{a}^{n}\left(M_{2}\right)$. Покажем, что $\varphi_{a}^{n}\left(M_{1}\right) \cap \varphi_{a}^{n}\left(M_{2}\right) \subset \varphi_{a}^{n}\left(M_{1} \cap M_{2}\right)$. Пусть $\xi=\varphi_{a}^{n}\left(x_{1}\right)=\varphi_{a}^{n}\left(x_{2}\right) \in$ $\varphi_{a}^{n}\left(M_{1}\right) \cap \varphi_{a}^{n}\left(M_{2}\right)$, где $x_{i} \in M_{i}, i=1,2$. Тогда $x_{1}-x_{2}=a y$, где $y \in A^{n}$. Так как $y \in \widehat{M_{1}+M_{2}}$, то ввиду замкнутости $M_{1}+M_{2}$ имеем $y \in M_{1}+M_{2}$. Пусть $y=y_{1}+y_{2}$, где $y_{i} \in M_{i}, i=1,2$. Тогда $x_{1}-a y_{1}=x_{2}+a y_{2} \in M_{1} \cap M_{2}$, $\varphi_{a}^{n}\left(x_{1}-a y_{1}\right)=\varphi_{a}^{n}\left(x_{1}\right)=\xi$. Таким образом, $\xi \in \varphi_{a}^{n}\left(M_{1} \cap M_{2}\right)$.

$\left(\right.$ б) $\Rightarrow(\mathrm{a})$. Пусть $x \in \widehat{M_{1}+M_{2}}$. Тогда $a x=x_{1}+x_{2}$, где $a \in A, x_{i} \in M_{i}$, $i=1,2$. Следовательно, $\varphi_{a}^{n}\left(x_{1}\right)=-\varphi_{a}^{n}\left(x_{2}\right)$, откуда $\varphi_{a}^{n}\left(x_{i}\right) \in \varphi_{a}^{n}\left(M_{1}\right) \cap \varphi_{a}^{n}\left(M_{2}\right)=$ $\varphi_{a}^{n}\left(M_{1} \cap M_{2}\right) . \quad$ Пусть $\varphi_{a}^{n}\left(x_{i}\right)=\varphi_{a}^{n}\left(y_{i}\right)$, где $y_{i} \in M_{1} \cap M_{2}, i=1,2$, тогда $x_{i}=y_{i}+a z_{i}$, где $z_{i} \in A^{n}, i=1,2$. Отсюда вытекает, что $z_{i} \in \widetilde{M}_{i}=M_{i}, i=1,2$. Следовательно, $a x=y_{1}+y_{2}+a z_{1}+a z_{2}$, откуда $a\left(x-z_{1}-z_{2}\right) \in M_{1} \cap M_{2}$. Так 
как $M_{1} \cap M_{2}$ замкнут, то $x-z_{1}-z_{2} \in M_{1} \cap M_{2}$. Значит, $x \in M_{1}+M_{2}$. Лемма доказана.

Завершим доказательство теоремы 5.3. Ввиду только что доказанной леммы для замкнутости $E_{1}(\omega)=F_{1}+F_{2}$ достаточно проверить, что $\varphi_{a}^{n}\left(F_{1} \cap F_{2}\right)=$ $\varphi_{a}^{n}\left(F_{1}\right) \cap \varphi_{a}^{n}\left(F_{2}\right)$. Но $F_{1} \cap F_{2}=B_{0}(\omega)$, поэтому в силу $\infty$-примитивности $\omega$

$$
\varphi_{a}^{n}\left(F_{1} \cap F_{2}\right)=\overline{B_{0}(\omega)}=B_{0}(\bar{\omega})=\varphi_{a}^{n}\left(F_{1}\right) \cap \varphi_{a}^{n}\left(F_{2}\right) .
$$

Теорема доказана.

Следующее предложение дает простой способ проверки $\infty$-примитивности $m$-вектора с четным $m$.

ПРЕДЛОЖЕНИЕ 5.3. Пусть $m \neq 2$ - четное число, $\omega-m$-вектор ранга 2 над кольиом Крулля $A$, причем $B_{0}(\omega)=0$. Тогда $\omega$-примитивен в том и только том случае, когда $\operatorname{div}\left(\frac{1}{2} \omega \wedge \omega\right)=1$.

ДокАЗАтЕльство. Пусть $\omega \infty$-примитивен. Так как $\operatorname{rank} \omega=2$, то $\omega=\widehat{x}_{1}+$ $\widehat{x}_{2}$, где $\widehat{x}_{i} \in \Lambda^{m}\left(A^{n}\right), i=1,2$. В силу теоремы $5.3 E_{1}(\omega)=X_{1} \oplus X_{2}$ - замкнутый модуль. Согласно [8; следствие 1] $d_{2 m}\left(E_{1}(\omega)\right)=1$. Но $\omega \wedge \omega=2 \widehat{x}_{1} \wedge \widehat{x}_{2}$, поэтому $\operatorname{div}\left(\frac{1}{2} \omega \wedge \omega\right)=d_{2 m}\left(E_{1}(\omega)\right)=1$.

Обратно, если $\operatorname{div}\left(\frac{1}{2} \omega \wedge \omega\right)=1$, то в силу [8; следствие предложения 1.1] свободный модуль $E_{1}(\omega)=X_{1} \oplus X_{2}$ замкнут. Ввиду леммы 5.3 это означает, что $\varphi_{a}^{n}\left(X_{1}\right) \cap \varphi_{a}^{n}\left(X_{2}\right)=0$ для любого $a \in A$. Следовательно, $\varphi_{a}^{n}\left(B_{0}(\omega)\right)=0$. Для $m$-векторов с $b_{0}(\omega)=0$ это означает $\infty$-примитивность.

СлеДСтВИЕ 5.3. Пусть $A$ - кольцо Крулля, $m \neq 2$ - четное число, $\omega \in$ $\Lambda^{m}\left(A^{n}\right)-m$-вектор $c \operatorname{div}\left(\frac{1}{2} \omega \wedge \omega\right)=1$. Для того чтоби $\operatorname{rank} \omega=2$, необходимо и достаточно, чтобь $B_{1}(\omega)=F_{1} \cup F_{2}$, әде $F_{1}, F_{2}$ - свободные подмодули в $A^{n}$ ранга $m$, а $E_{1}(\omega)$ бъл замкнут.

\section{Список литературы}

1. Plucker J. Neu Geometrie des Raumes gegrundet auf die gerade Linie als Raumelement. Leipzig, 1868-1869.

2. Segre S. Mehrdimensionale Raume. Encyklopadie der Mathematischen Wissenschaften. III. C7, 1915.

3. Martinet J. Sur les singularités des formes differentielles // Ann. Inst. Fourier. 1970. V. 20. № 1. P. 95-178.

4. Busemann H., Glassco D. E. Irreducible sums of simple multivectors // Pacific J. Math. 1973. V. 49. № 4. P. 13-32.

5. Бурбаки H. Алгебра. Алгебраические структуры. Линейная и полилинейная алгебра. М.: Физматгиз, 1962.

6. Клейнер Г. Б. Поливекторы ранга 2 // УМН. 1974. Т. 29. № 3. С. 204-208.

7. Towber J. Complete reducibility in exterior algebra over free modules // J. Algebra. 1968. V. 10. № 3. P. 299-309.

8. Клейнер Г. Б. О плюккеровых свойствах колец // Матем. сб. 1971. Т. 84.№ 4 . C. $526-536$.

9. Клейнер Г. Б. Замечание о детерминантных последовательностях // Труды объединения математических кафедр педагогических институтов центральной зоны РСФСР. Т. 1. № 1-2, 1972. C. 109-114. 
10. Lim M. J. S. Rank $k$ Grassman products // Pacific J. Math. 1969. V. 29. № 2. P. 367-374.

11. Котлярский М. А. Два замечания о плюккеровых кольцах // УМН. 1975. Т. 30. № 2 . C. 213 .

Центральный экономико-математический институт РАН, г. Москва

Поступила в редакцию

E-mail: kleiner@cemi.rssi.ru 08.11 .2000 